First results examining the compatibility of RMP ELM suppression with the radiating divertor in DIII-D

T. W. Petrie, T. E. Evans, M. E. Fenstermacher, S. Mordijck,

N. H. Brooks, J. R. Ferron, B. Hudson, A. W. Hyatt, C. J. Lasnier, A. W. Leonard, T. C. Luce, R. A. Moyer, P. A. Politzer, M. J. Schaffer, P. B. Snyder, J. G. Watkins

May 21, 2010

19th PSI Conference

San Diego, CA, United States

May 24, 2010 through May 28, 2010 
This document was prepared as an account of work sponsored by an agency of the United States government. Neither the United States government nor Lawrence Livermore National Security, LLC, nor any of their employees makes any warranty, expressed or implied, or assumes any legal liability or responsibility for the accuracy, completeness, or usefulness of any information, apparatus, product, or process disclosed, or represents that its use would not infringe privately owned rights. Reference herein to any specific commercial product, process, or service by trade name, trademark, manufacturer, or otherwise does not necessarily constitute or imply its endorsement, recommendation, or favoring by the United States government or Lawrence Livermore National Security, LLC. The views and opinions of authors expressed herein do not necessarily state or reflect those of the United States government or Lawrence Livermore National Security, LLC, and shall not be used for advertising or product endorsement purposes. 


\title{
First results examining the compatibility of RMP ELM suppression with the radiating divertor in DIII-D
}

\author{
T.W.Petrie $\mathrm{a}^{\mathrm{*}}$, T.E. Evans ${ }^{\mathrm{a}}$, M.E. Fenstermacher ${ }^{\mathrm{b}}$, S. Mordijck ${ }^{\mathrm{c}}$, N.H. Brooks ${ }^{\mathrm{a}}$, \\ J.R. Ferron ${ }^{\mathrm{a}}$, B. Hudson ${ }^{\text {d }}$, A.W. Hyatt ${ }^{\mathrm{a}}$, C.J. Lasnier ${ }^{\mathrm{b}}$, A.W. Leonard ${ }^{\mathrm{a}}$, T.C. Luce ${ }^{\mathrm{a}}$, \\ R.A. Moyerc, P.A. Politzer ${ }^{\text {a }}$, M.J. Schaffer ${ }^{\mathrm{a}}$, P.B. Snydera ${ }^{\mathrm{a}}$, and J.G. Watkins ${ }^{\mathrm{e}}$

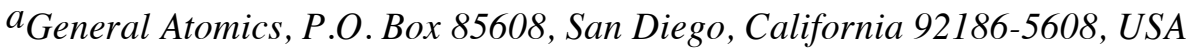

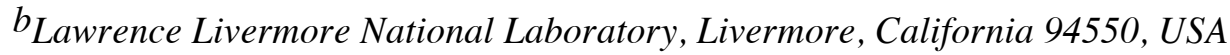 \\ ${ }^{c}$ University of California San Diego, San Diego, California 92093, USA

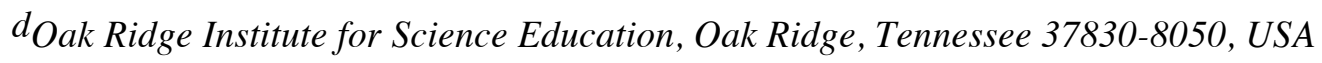 \\ e Sandia National Laboratories, Albuquerque, New Mexico 87185, USA
}

\begin{abstract}
We report on recent DIII-D experiments that integrate edge localized mode (ELM) suppression using resonant magnetic perturbations (RMPs) with divertor heat flux reduction under radiating divertor conditions. Our results illustrate the limitations in maintaining ELM suppression at gas puffing levels that were representative of good puff-and-pump operation in previous experiments without RMP. The electron pressure gradient in the pedestal $(\nabla P e)$ increased steadily during gas puffing and ELMs returned once $\nabla P e$ reached values consistent with the peeling-ballooning stability limit, as determined by ELITE edge stability analysis. Even with this return of ELMs, a radiating divertor with RMP generated higher levels of total radiated power $(\sim 40 \%)$ than comparable standard ELMing discharges without RMP at the same density. Differences in the accumulation of the seed argon in the core plasma between RMP and non-RMP during puff and pump were less than $20 \%$.
\end{abstract}


JNM keywords: Divertor Materials (D0500), Impurities (I0100), Plasma-Materials Interaction (P0500), Plasma Properties (P0600)

PSI-19 keywords: Argon, DIII-D, Divertor, Edge pedestal, ELM, Particle control PACS: 52.25.Vy, 52.55.Fa, 52.55.Rk, 89.30.Jj

*Corresponding and presenting author address: General Atomics, P.O. Box 85608, San Diego, California 92186-5608, USA

*Corresponding and presenting authore-mail:petrie@fusion.gat.com 


\section{Introduction}

The impulsive heat loads to the wall material at the divertor targets during ELMs result in severe erosion that significantly shortens its lifetime [1]. Recent investigations, however, have demonstrated that such damaging ELMs can be suppressed by applying resonant magnetic perturbations (RMPs) to the pedestal region of the plasma [2,3]. While studies to improve understanding of the underlying physics are ongoing, the usefulness of RMPs in suppressing ELMs presents a plausible option for mitigating ELM-damage to divertor surfaces.

Despite the fact that RMPs effectively eliminate impulsive heat damage from ELMs, the steady heating component at the divertor targets for highly powered tokamaks like ITER can still be unacceptably high. In previous studies, radiating divertor solutions were effective in reducing and controlling the steady flow of heat to the divertor targets [4-7]. In DIII-D, the "puff-and-pump" scenario was found to be effective in reducing the overall power load at the divertor targets with little degradation of H-mode plasma properties. (In the puff-and-pump approach, "seed" impurities are injected into the private flux region (PFR) and restrained from penetrating the plasma core by a combination of deuterium gas injection upstream and active particle exhaust at the divertor targets.)

Separately, the RMP technique addresses the impulsive heat load from the ELMs and puff-and-pump addresses the steady heat load. It is unclear, however, whether RMP ELM suppression can be successfully merged with a radiating divertor solution. In this paper we examine this compatibility issue, particularly from the standpoints (1) of maintaining ELM suppression during gas injection and (2) of evaluating radiating divertor behavior with RMP. The experimental arrangement and methodology are described in Sec. II. In Sec. III we present our results and we discuss them in Sec. IV. 


\section{Experimental setup}

The poloidal cross-section of the lower single-null ( $\mathrm{SN}$ ) configuration used in this study is shown in Fig. 1. In-vessel, active pumping of the injected deuterium $\left(D_{2}\right)$ and argon (Ar) gases is done by a single cryo-pump located in the lower divertor plenum. The outer divertor strike point radius (Rosp) is situated adjacent to the entrance of the lower divertor plenum. Argon is injected directly into the PFR, while $\mathrm{D}_{2}$ is injected into the crown of the lower $\mathrm{SN}$ configuration in order to increase the deuterium ion flow toward the lower divertor pump. Argon is used as the seeded impurity in this experiment because it radiates effectively at the temperatures prevailing in both the divertor and pedestal regions of DIII-D H-mode plasmas and has a relatively short, ionization mean free path. Carbon, generated by erosion of the graphite armor, is the dominant intrinsic impurity in DIII-D discharges. The plasmas in this study are characterized by: $H_{98(y, 2)} \approx 0.9-1.2, \quad q_{95}=3.5, \quad \beta_{N} \approx 2, \quad \bar{n}_{e} / n_{e G} \approx 0.3-0.7$, $P_{\mathrm{RAD}} / P_{\mathrm{INJ}} \approx 0.3-0.8, Z_{\text {eff }} \approx 2$, and the direction of the ion $\boldsymbol{B} \times \nabla B$ drift is toward the X-point. For the RMP H-mode discharges discussed in this study, we place the outer strike point at the radial position that yields maximum pumping.

DIII-D has two off-axis rows of six internal coils each, the "I-coil", that are used for ELM suppression and mitigation experiments in an $n=3$ magnetic configuration [8]. The experiments in this paper employed the I-coil with $n=3,60^{\circ}$ phasing in even parity, that is, currents of the same polarity in coils above and below the midplane at the same toroidal location (i.e., up-down symmetric). For the maximum coil current, this results in a perturbation strength of $\delta b_{r} \sim 6.5 \times 10^{-4} \mathrm{~T}$ at $\Psi_{N}=0.95$ [3].

The I-coil current is set to near its maximum value $(5.8 \mathrm{kA})$, and ELM suppression is obtained for an edge $q_{95}$ in the range 3.25 - 3.65 . 


\section{Results}

\section{A. ELM-suppression during gas puffing}

Figure 2 shows that RMP-induced, ELM suppression is diminished and ultimately lost even at modest rates of deuterium and argon injection [Figs. 2(IIa), 2(IIIa)]. The activation of the I-coils at $t=2.0 \mathrm{~s}$ results in an immediate decrease in the pedestal density $n_{\mathrm{PED}}: n_{e} / n_{e \mathrm{G}}=0.5$ and 0.3 at $t=1.9 \mathrm{~s}$ (pre-activation of the I-coil) and $2.7 \mathrm{~s}$ (postactivation of the I-coil), respectively. The ELMs are suppressed within $200 \mathrm{~ms}$ of I-coil activation. Deuterium and argon injection begin at $2.8 \mathrm{~s}$ and $3.2 \mathrm{~s}$, respectively. As with ELMing H-mode plasmas without RMP, higher $\Gamma_{\mathrm{D} 2}$ produces a more rapid increase in $n_{P E D}$ [Figs. 2(Ib)-2(IIIb)] and a measurable drop in pedestal electron temperature $T_{\mathrm{PED}}$ [Figs. 2(Ic)-2(IIIc)]. The energy confinement factor, $H_{98(y, 2)}$, is 1.2 before the I-coil is activated, drops to $\approx 0.9$ following activation, and is constant for the rest of the discharge [Figs. 2(Id)-2(IIId)]. After the initial drop in the edge electron pressure gradient $\nabla P_{e}$ [Figs. 2(Ie)-2(IIIe)] following I-coil activation, $\nabla P_{e}$ partially recovers during subsequent gas puffing. The higher the deuterium gas puff rate, the sooner ELMs reappear. The range in $n_{\mathrm{PED}}$ for ELM-suppressed operation is relatively small for these puff-and-pump plasmas.

The electron collisionality in the pedestal $\left(v_{e}^{*}\right)$ and the maximum gradient in the pedestal electron pressure $\left(\nabla P_{e-\mathrm{MAX}}\right)$ are plotted versus $n_{\mathrm{PED}}$ for three phases of these H-mode discharges: (1) ELMing, (2) transition ELM behavior, and (3) ELM-suppressed (Fig. 3). While the ELMing and ELM-suppressed phases are self-explanatory, the transitional phase refers to times where sporadic ELMing is occurring. During the ELMing phase before the Icoil is activated, the average $n_{\mathrm{PED}}$ is $\approx 0.39 \times 10^{20} \mathrm{~m}^{-3}$. Shortly after I-coil activation, ELMsuppression is observed when $n_{\mathrm{PED}}$ is in the range $(0.17-0.25) \times 10^{20} \mathrm{~m}^{-3}$, a transition 
interval for $n_{\mathrm{PED}} \approx(0.24-0.30) \times 10^{20} \mathrm{~m}^{-3}$, and finally for $n_{\mathrm{PED}}>0.30 \times 10^{20} \mathrm{~m}^{-3}$, a return to the "pure" ELMing regime. Figure 3 shows that even for modest increases in the gradient of the pedestal pressure, ELM-suppressed plasmas can transition to solidly ELMing H-mode discharges. Analysis using the ELITE edge plasma stability code [9] suggests that peelingballooning mode instabilities trigger the onset of these type-1 ELMs. Hence, it is not surprising that an increase in pedestal $\nabla P_{e}$ may promote the re-emergence of ELMing.

While the reappearance of ELMing can be associated with increases in pedestal $\nabla P_{e}$, the precise role of pedestal electron collisionality in the reappearance of ELMing is less clear. ELM-suppression is observed for plasmas with $v_{e}^{*}<0.3$, the transition from ELM-suppressed to ELMing with $v_{e}^{*} \approx 0.3-0.4$, and the ELMing regime with $v_{e}^{*}>0.45$.

\section{B. Argon accumulation in the main plasma}

Differences in argon accumulation inside the main plasma between RMP and similar nonRMP ELMing H-mode plasmas were less than 20\% [Fig. 4]. As with the non-RMP cases, the core concentration of argon in the RMP cases decreases with increasing $\Gamma_{\mathrm{D} 2}$. This suggests that many of the physical processes detailed in UEDGE [10] fluid transport modeling reported previously for non-RMP radiating divertor plasmas [7] may also be important in these corresponding RMP cases, e.g., the importance of particle drifts in "fueling" the core plasma. This is a key point, because the extensive studies in optimizing performance of radiating divertor plasmas in non-RMP cases provide direction as to how radiating divertor cases with $R M P$ might be optimized. The return of Type-1 ELMing activity at the higher gas puff rates (or higher $n_{\mathrm{PED}}$ ) in RMP plasmas may be responsible for the similarity in argon impurity accumulation in the main plasma. UEDGE modeling of these RMP plasmas is underway. 


\section{Radiating divertor with RMP}

When applied to puff-and-pump plasmas, RMP enhances the plasma radiated power. A comparison of similarly prepared H-mode plasmas with and without RMP (but with the same pedestal density) shows that the radiated power was $\approx 40 \%$ higher in the RMP case (Fig. 5). The ratio of radiated power to input power $\left(P_{\mathrm{RAD}} / P_{\mathrm{IN}}\right)$ was significantly greater for puffand-pump cases than for the corresponding standard ELMing H-mode plasmas with similar power input and pedestal density. Approximately one-third of this increase occurred in the SOL and divertor regions and two-thirds of this increase in the main plasma. The increase in the SOL and divertor radiated power was largely due to the higher puffing rates of deuterium (and argon "seed" impurity) gas puffing required to maintain the pedestal density (i.e., $n_{\mathrm{PED}}$ $n_{\mathrm{PED}} \approx 0.39 \times 10^{20} \mathrm{~m}^{-3}$ ) after the I-coil is activated, and this, in turn, produced a thicker SOL density and lower plasma temperatures in both the plasma edge and SOL/divertor that favored higher radiated power. The increase in the radiated power in the main plasma was largely due to the accumulation of argon and a $10-15 \%$ reduction in $T_{\mathrm{PED}}$. While $H_{98(y, 2)}$ was reduced $\approx 25 \%$ after the I-coil was activated, the energy confinement time during subsequent deuterium and argon puffing continued to be representative of a good H-mode [i.e., $H_{98(y, 2)} \approx 0.9$ ] and was insensitive to changes in $n_{\mathrm{PED}}$. While ELMing re-appeared during the gas puffing phases with higher $\Gamma_{\mathrm{D} 2}$, we found that the peak heat flux deposited at the inner divertor targets during an ELM event was $\approx 30-40 \%(\approx 50-60 \%)$ lower than its precoil levels for the cases $n_{\mathrm{PED}} \approx 0.39 \times 10^{20} \mathrm{~m}^{-3}\left(\approx 0.50 \times 10^{20} \mathrm{~m}^{-3}\right)$, respectively.

\section{Discussion}

ELMing activity in these plasmas ceased shortly after the activation of the I-coil, and both $n_{\mathrm{PED}}$ and $\nabla P_{e}$ in the pedestal were reduced $\approx 50 \%$. When deuterium and argon gas were 
injected, both $n_{\mathrm{PED}}$ and $\nabla P_{e}$ at the edge were increased. However, when ELMing reemerged, $n_{\mathrm{PED}}$ had only recovered to $\approx 70 \%$ and $\nabla P_{e}$ only $\approx 80 \%$ of their pre-activation values. Because ELITE code analysis suggests that peeling-ballooning mode instabilities may be triggering the re-appearance of ELMs, the observed increases in $\nabla P_{e}$ during "recovery" may be associated with making the pedestal more susceptible to ELMs. Figure 3 suggests that the "headroom" in pedestal $\nabla P_{e}$ between ELM-suppressed and the ELMing conditions is relatively small for the plasmas under investigation.

Successful ELM suppression by RMP clearly puts a limit on $\Gamma_{\mathrm{D} 2}$ (and $\Gamma_{\mathrm{AR}}$ ) that is available for the puff-and-pump operation. Previous studies of the puff-and-pump approach at DIII-D have shown that higher $\Gamma_{\mathrm{D} 2}$ leads to better screening of the seed impurity from the main plasma [6]. The "best" puff-and-pump results for the plasmas described here would require $\Gamma_{\mathrm{D} 2} \sim 12-13 \mathrm{~Pa} \mathrm{~m} / \mathrm{s}$, which is considered above the maximum allowed $\Gamma_{\mathrm{D} 2}$ for complete ELM-suppression in similar plasmas described here (i.e. $<3.5 \mathrm{~Pa} \mathrm{~m}^{3} / \mathrm{s}$ ). These results highlight the challenges for future devices in combining RMP-based ELM suppression with optimal puff-and-pump radiating divertor.

Two ways that might extend the range in ELM-suppression are worth considering. The first approach focuses on inhibiting the buildup of pedestal $\nabla P_{e}$, since our results suggest that the increase in pedestal $\nabla P_{e}$ enhances the chance of triggering an ELM. Two possibilities to consider here are (1) increasing the I-coil current during gas puffing and (2) directing ECH absorption in the pedestal. In the former, sufficient power supplies for the I-coil is the crucial consideration, although increases in I-coil current that would degrade energy confinement and plasma performance is another downside. In the latter, ECH applied to plasma edge would enhance particle transport near the maximum in $\nabla P_{e}$ and presumably inhibit the building in $\nabla P_{e}$

The second general approach is based on enhancing the particle exhaust by exploiting 
what we learned in previous puff-and-pump experiments about how particle drifts affect pumping effectiveness. In the plasma discussed here, particle pumping was done only on the outer divertor leg with the ion $\boldsymbol{B} \times \nabla B$ drift directed toward the $\mathrm{X}$-point. While this arrangement has been successful in suppressing ELMs, compared with the other pumping configurations available on DIII-D, this arrangement is least effective in controlling particle inventory and fueling of the main plasma. Based on previous work in non-RMP radiating divertors [7], the most effective way to apply RMP ELM suppression in puff-and-pump plasmas is to maximize divertor pumping and operate with the ion $\boldsymbol{B} \times \nabla B$ drift directed away from the X-point. For DIII-D, this would mean SN operation in the closed upper divertor which has much stronger pumping, i.e., two cryo-pumps available, with the ion $\boldsymbol{B} \times \nabla B$ drift directed away from the X-point. A discussion of the technical issues that we encountered in executing these ideas (e.g., avoiding 2/1 locked modes at low density) will be explored in a future paper.

While primary interest in RMP has been largely directed toward ELM-suppression, we found that the puff-and-pump radiating divertor, augmented with RMP, yielded significantly higher radiative fractions than the standard ELMing $\mathrm{H}$-mode plasma at the same $n_{\mathrm{PED}}$. At a slightly higher pedestal density (i.e., $n_{\mathrm{PED}} \approx 0.50 \times 10^{20} \mathrm{~m}^{-3}$ ), the fraction of radiated power increased further to 0.75 . The energy confinement factor $H_{98(y, 2)}$ was insensitive to the higher gas puff rate that this required. Even though ELMs re-appeared during the gas puffing phase for several cases discussed in Sec. IIIC, we found that the peak heat flux deposited at the inner divertor targets during an ELM event could be significantly reduced with a combination of RMP and gas injection. Our results indicate that ELM mitigation at higher density and gas puffing rates may be more readily-attained than complete ELM suppression. 


\section{Acknowledgment}

This work was supported by the U.S. Department of Energy under DE-FC02-04ER54698,

DE-AC52-07NA27344(LLNL)， DE-FG02-07ER54917， DE-AC05-06OR23100, DE-AC04-94AL85000, and DE-FG02-05ER54809 . 


\section{References}

[1] G. Federici, et al., Plasma Phys. Control. Fusion 45 (2003) 1523.

[2] T.E. Evans, et al., Phys. rev. Lett. 92 (2004) 235003.

[3] T.E. Evans, et al., Nucl. Fusion 48 (2008) 024002.

[4] J. Rapp, et al., Nucl. Fusion 44 (2004) 312.

[5] A. Kallenbach, et al., J. Nucl. Mater. 337-339 (2005) 732.

[6] T.W. Petrie, et al., J. Nucl. Mater. 363-365 (2007) 416.

[7] T.W. Petrie, et al., Nucl. Fusion 48 (2008) 045010.

[8] T.E. Evans, et al., Phys. Plasmas 13 (2006) 056121.

[9] P.B. Snyder, H.R. Wilson, et al., Phys. Plasmas 9 (2002) 2037.

[10] T.D. Rognlien, et al., J. Nucl. Mater. 196-198 (1992) 347. 


\section{Figure Caption}

Fig. 1: MHD cross-section of the lower single-null configuration with the gas injection, divertor pumping plenum, and RMP I-coil locations superimposed.

Fig. 2: The re-emergence of ELMing activity for three discrete values of $\Gamma_{\mathrm{D} 2}: 0,5$, and $10 \mathrm{~Pa} \mathrm{~m}^{3} / \mathrm{s}$ in columns I, II, and III, respectively. (a) Deuterium recycling $D_{\alpha}$, (b) $n_{\mathrm{PED}}$, (c) $T_{\mathrm{PED}}$, (d) $H_{98(y, 2)}$, and (e) $\nabla P_{e}$. Note that the argon (blue) and the deuterium (yellow) boxes in (b) represent only their injection times and are not to scale. $\Gamma_{\mathrm{Ar}}=0.05 \mathrm{~Pa} \mathrm{~m} / \mathrm{s}$ and $\mathrm{I}-$ coil $=5.8 \mathrm{kA}$ in each case.

Fig. 3: Electron collisionality and the maximum pressure gradient in the pedestal are plotted versus $n_{\mathrm{PED}}$.

Fig. 4: Argon accumulation in the core plasma $\left(n_{\mathrm{Ar}}\right)$ as a function of $\Gamma_{\mathrm{D} 2}$, in both RMP and non-RMP radiating divertor discharges.

Fig. 5 (a) $P_{\mathrm{RAD}} / P_{\mathrm{IN}}$, (b) $H_{98(y, 2)}$, and (c) $n_{\mathrm{PED}}$ are plotted versus $\Gamma_{\mathrm{D} 2} \cdot \Gamma_{\mathrm{Ar}}$ is fixed at $0.05 \mathrm{~Pa} \mathrm{~m} 3 / \mathrm{s}$. Data with RMP are shown for a range in $\Gamma_{\mathrm{D} 2}$ (solid circle). The reference case of ELMing without RMP is shown for $\Gamma_{\mathrm{D} 2}=0$ (solid box). Plasma parameters: $I_{p}=1.43 \mathrm{MA}, q_{95}=3.5$, and $P_{\mathrm{IN}}=5.4-6.5 \mathrm{MW}$. 


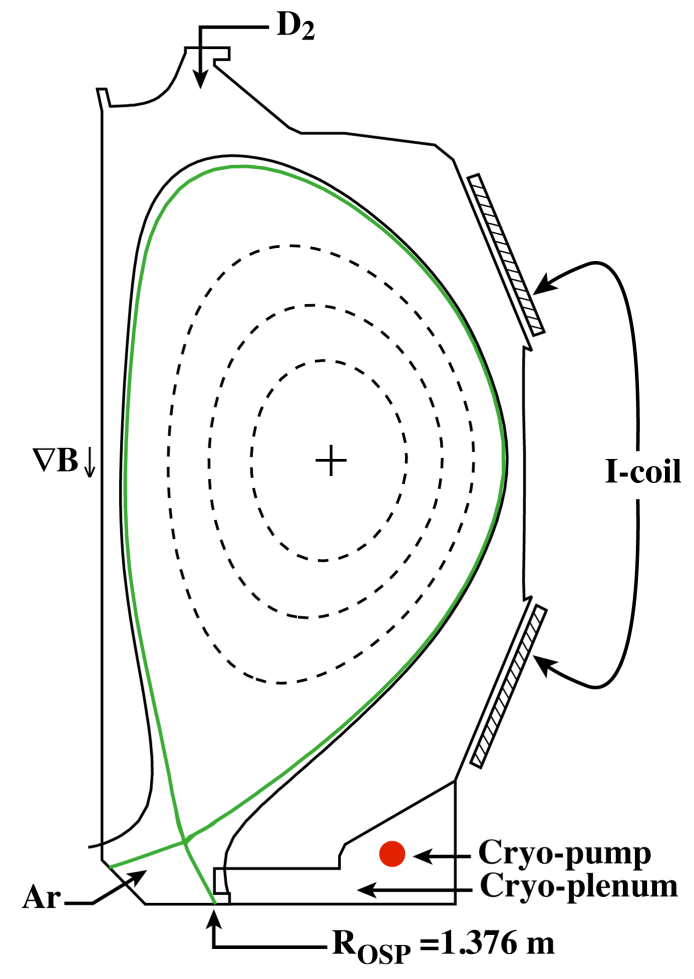

Figure 1 


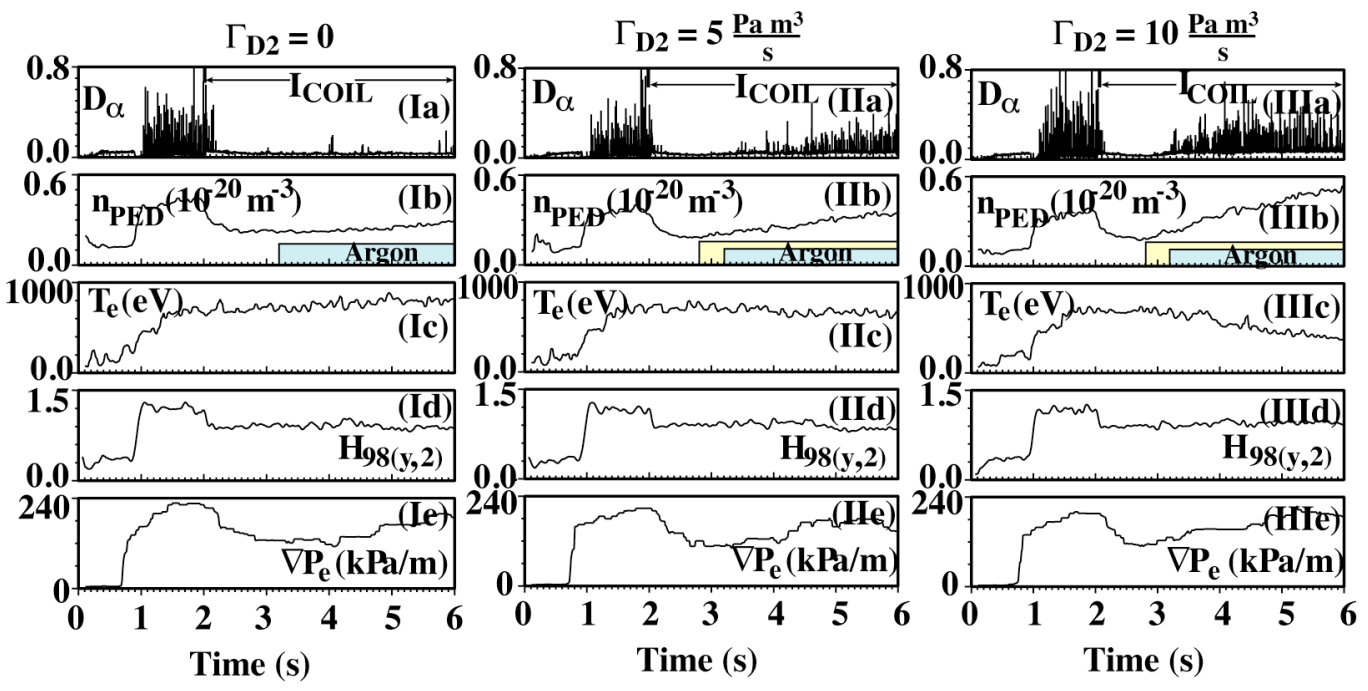

Figure 2 


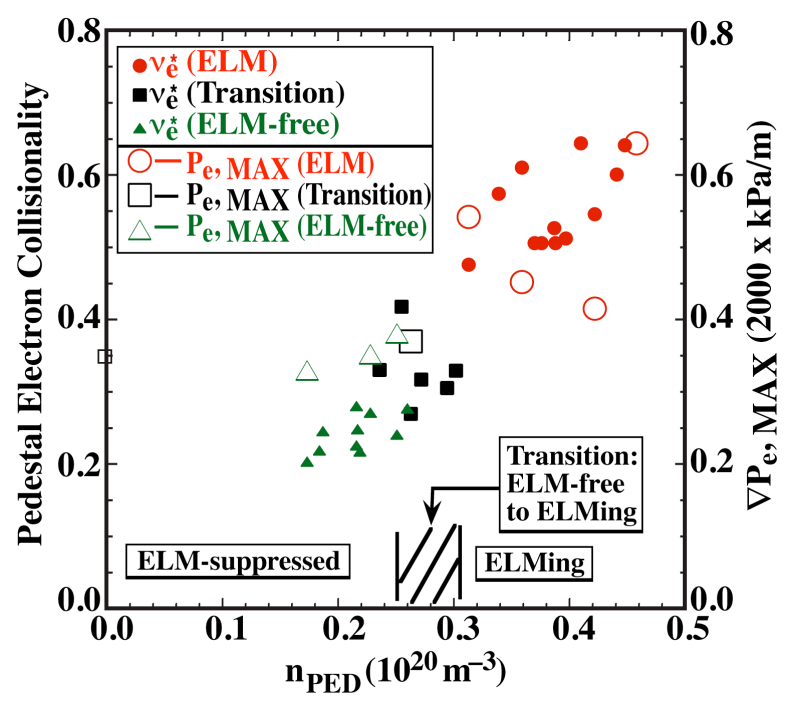

Figure 3 


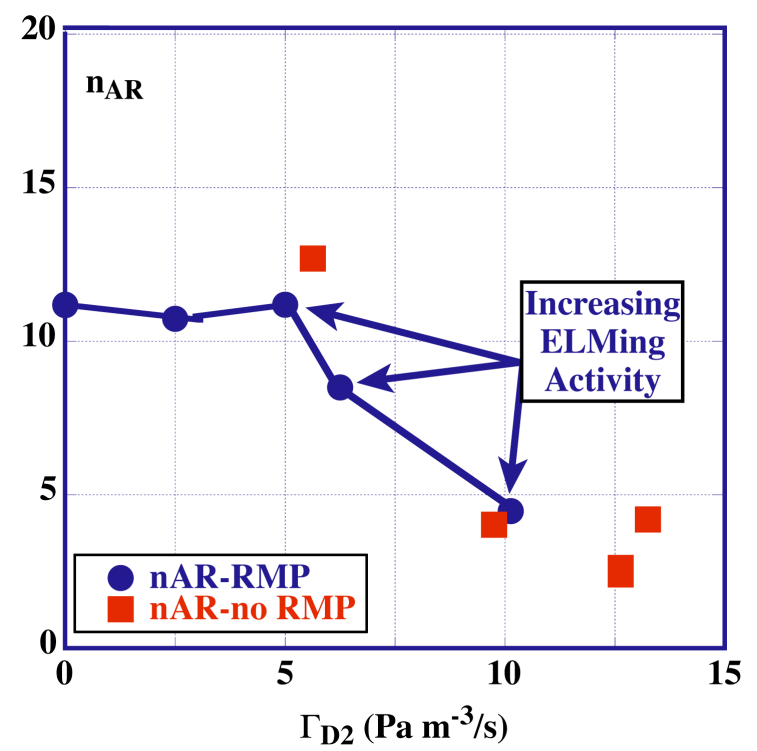

Figure 4 

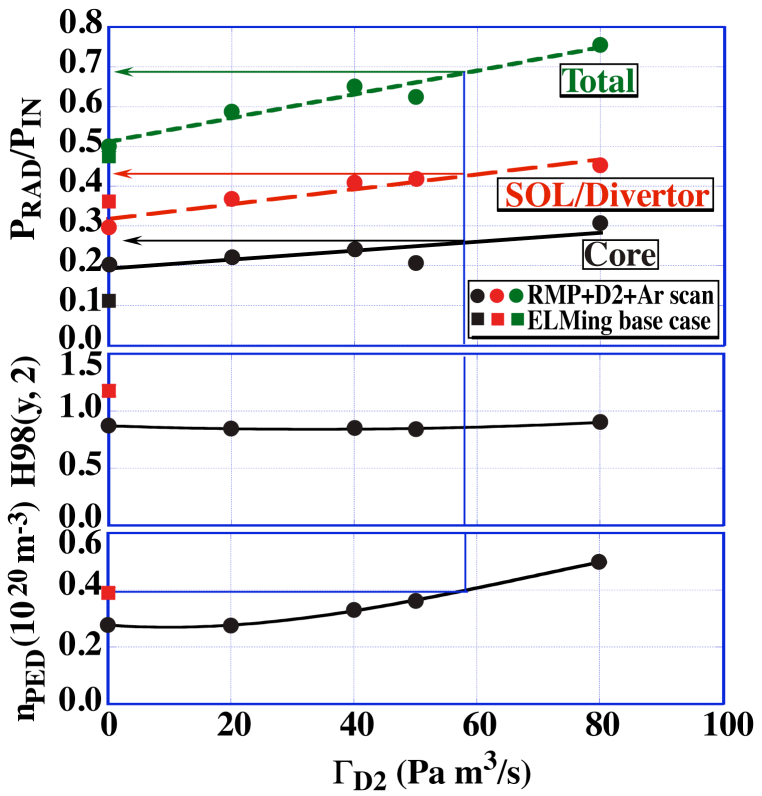

Figure 5 